Does the left hair part look better (or worse) than the right?

Jeremy A. Frimer, University of Winnipeg

January 8, 2018

Word Count: 3592

in press in Social Psychological and Personality Science

Corresponding Author: Jeremy A. Frimer, Department of Psychology, University of Winnipeg,

515 Portage Avenue, Winnipeg MB, Canada, R3B 2E9, j.frimer@uwinnipeg.ca

Acknowledgments: This work was supported by a grant from the Social Sciences and

Humanities Research Council of Canada to J. A. Frimer [grant number 435-2013-0589].

Open Science Practices: The data sets and stimuli for this paper are publicly available at http://osf.io/hmv8n. All methods are fully described in the text. 


\begin{abstract}
This paper provides the first experimental tests of whether the side of the head on which a person parts his/her hair affects the person's appearance and perceived character. The popular culture view is that the left hair part makes a person appear more competent and masculine, and the right part warmer and more feminine. Participants judged the appearance of a portrait with a hair part on one side, or a digitally altered version in which the hair part was reversed (while the face remained the same). Three studies (total $N=3819$ ) found that hair part does not alter appearance. The null results generalized to male and female models, smiling and neutral expressions, and hair parts flowing with or against the hair whorl. The popular belief that hair part alters one's appearance may either be a myth of underspecified.
\end{abstract}

Keywords: hair part, attractiveness, facial asymmetry, warmth, competence 


\section{Does the left hair part look better (or worse) than the right?}

In the 1978 Superman movie, Christopher Reeve played the feeble character Clark Kent, who parted his hair on his right. When Kent emerged as the powerful Superman, his hair was parted on the left. The next year, U.S. President Jimmy Carter made the same hair part switch, from right to left (Murphy, 1999). And in 2008, Hillary Clinton made the opposite switch, from left to right (Ellerson, 2008). These examples may reflect a circulating belief in the population that a person's hair part changes a person's appearance and perceived character strengths.

Fashion websites (e.g., Female Magazine, 2013), magazines such as The Atlantic (Murphy, 1999) and The New Yorker (Schillinger, 2001), and podcasts like Radiolab (Abumrad \& Krulwich, n.d.) offered advice about parting the hair on the left to look competent or masculine and on the right to look warm or feminine. The belief appears to have been reified by a nuclear physicist and cultural anthropologist team, who developed what they call the Hair Part Theory (Walter \& Walter, 1999), and spun off a company that sells non-reversing mirrors.

Evidence supporting the view that hair part orientation affects appearance has been correlational, unsystematic, and inconsistent. For instance, Walter and Walter (1999) reported a larger tally of U.S. Presidents that parted their hair on the left than the right, with the former set appearing to have higher approval ratings than the latter. They provided no inferential tests, and did not address the reverse-causal or third variable problems. Moreover, their data have not been subjected to peer review. Rigorous scientific investigation of this matter has been conspicuously absent, and psychologists almost entirely reticent.

One prominent psychologist, M. Nicholls, speculated about why hair part might change a person's appearance, without claiming that it would (Abumrad \& Krulwich, n.d.). If the distinct nature of the hair part directs observers' attention to the adjacent side of the face, then hair parts 
could change a person's overall appearance when the model is expressing positive emotion. This is because, as a result of cerebral hemispheric lateralization, the left side of the face is more emotionally expressive than the right side (Borod, Kent, Koff, Martin, \& Alpert, 1988; Borod, Koff, \& White, 1983; Campbell, 1978; Ekman, Hager, \& Friesen, 1981; Indersmitten \& Gur, 2003; Nicholls, Ellis, Clement, \& Yoshin, 2004; Rubin \& Rubin, 1980; Sackeim \& Gur, 1978; Sackheim, Gur, \& Saucy, 1978; for a meta-analysis, see Skinner, \& Mullen, 1991). This leads to the prediction that left hair part will improve the overall appearance of a person, especially when the person is showing positive emotion. An alternative possibility is the null: that the side of the head on which one parts their hair has little or no effect on their overall appearance. The goal of the present research was to provide the first systematic tests of whether hair part influences attractiveness and perceived character.

\section{Study 1}

Study 1 experimentally tested whether hair part alters appearance along several traits. Popular culture and Hair Part Theory have speculated about the moderating role of gender. I thus included both a male and female model. The theory that the left side of the face is more emotionally expressive than the right gave rise to the prediction that facial expression might moderate the effect of hair part on appearance. I thus included both smiling and neutral expressions. And I included four traits: overall appearance, warmth and competence (the primary dimensions of social judgment; Abele \& Wojciszke, 2013; Cuddy, Fiske, \& Glick, 2007; Rosenberg, Nelson, \& Vivekananthan, 1968; Trapnell \& Wiggins, 1990), and femininity/masculinity.

\section{Method}


Participants. Sample size was determined before any data analysis. To determine a sample size, I required an effect size estimate. Unfortunately, no research has directly tested whether hair part orientation influences a model's appearance. However, if hair part directs attention to its adjacent side of the face, then the asymmetry in emotional expressiveness might provide an appropriate effect size estimate. Indersmitten and Gur (2003) reported an asymmetry effect size of $d=0.55$ when participants viewed left-left or right-right composites of smiling faces. Insofar as split half composites would double the available information, I reasoned that the hair part effect size might be approximately half of the reported one, $d=0.28$. I recruited a conservatively large sample to bias the study in favor of detecting a small hair part effect, if one exists. Thus, rather than using the conventional $80 \%$ power, I sought $99.99 \%$ power, necessitating a sample of 1648 .

I report all measures, manipulations, and exclusions in all studies. I recruited $N=1654$ participants from Amazon's Mechanical Turk (AMT; each received \$0.30). The sample spanned much of the lifespan (18-81 years) with $M=35.8(S D=11.7)$, and was gender-balanced (55\% male, $45 \%$ female). Most participants (81\%) were from the U.S., with minorities from India (14\%), Canada (1\%), and 4\% from 29 other countries combined.

Procedure. Participants viewed four portrait images. For each participant, the hair part in all four images was on the left or on the right (randomly assigned between-subjects; $n$ s $=843 \&$ 810, respectively). The images were from the Chicago Faces Database (Ma, Correll, \& Wittenbrink, 2015; see Figure 1). They depicted a White female or White male model, with either a neutral or a smiling expression. Each image was in black-and-white and 400 pixels tall. I used black-and-white because it was more amenable to image preparation than color images. The four images ( 2 models $\times 2$ expressions $)$ appeared in counterbalanced order. All of the original 
images had the hair part on the model's left. I digitally altered the hair part to create the right part images (see Figure 2).

Figure 1. Experimental stimuli (Study 1). Participants saw four pictures of a male and female model with a neutral or smiling expression. I randomly assigned each participant to see the hair part on the left or the right in all four portraits. The order of presentation was counterbalanced.

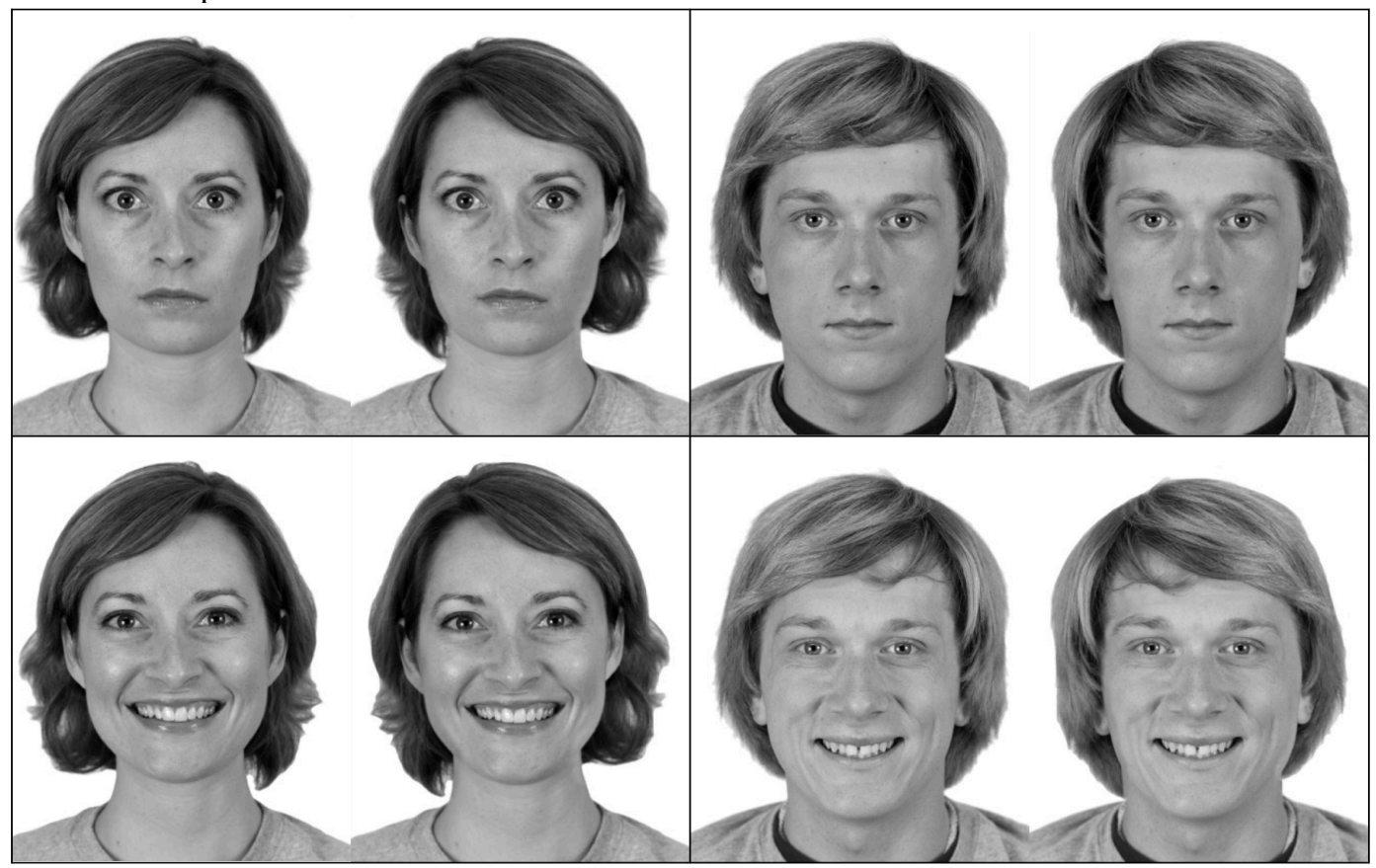

Figure 2. The digital process that reversed the hair part while leaving the face and its expression unchanged.

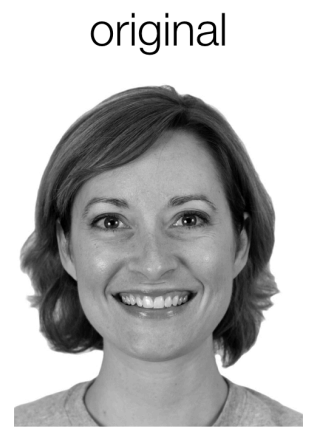

remove face

reverse

superimpose

blend

Image preparation. A critical design feature was portrait preparation. Simply flipping a

portrait horizontally would be suboptimal because it would flip the facial expression along with the hair, introducing a confound. Ideally, the stimuli would hold the face and its expression constant, while horizontally flipping the hair part. To achieve this, I digitally altered the original 
images by horizontally flipping the hair without changing the facial features. Figure 2 illustrates the image preparation process.

Appearance. Participants rated the models' appearance on a scale from 0 to 10 on four traits. The question asked, "How does this person look?" The trait rating scales were bipolar: (a) ugly (0) - attractive (10), (b) cold (0) - warm (10), (c) incompetent (0) - competent (10), and (d) masculine (0) - feminine (10). For the male model, I reverse-scored the final item and refer to it as "gender-consistent".

\section{Results}

Figure 3 shows how hair part orientation had a negligible effect on the rated appearance and character of the portrayed models. I systematically tested whether hair part changes the appearance of a person, and whether any such changed depends on whether the model is male or female, whether the models is smiling or has a neutral expression, and whether it depends on the perceived trait. Table 1 presents the results of a 2 (Model: Male, Female) $\times 2$ (Expression: Neutral, Smiling) $\times 4$ (Trait: Attractive, Warm, Competent, Gender-Consistent $) \times 2$ (Part Direction: Left, Right) Mixed Model ANOVA (with the first three factors within-subjects and the final factor between-subjects). Hair part direction did not alter the appearance of the models, regardless of the model's gender, expression, or trait, or any combination thereof. Hair part did not alter the impression on any of the 16 variables (see Tables $2 \& 3$ ). Given the null findings, I used Bayesian Analysis to estimate the posterior probability of the null (Masson, 2011), and found that all effects involving hair part had $>96 \%$ probability of the null being true given the data. 
Figure 3. Rating of the female and male models with neutral or smiling expressions, and with the hair part on their left or right. In spite of the large sample size and statistical power, analyses failed to detect any significant variance associated with hair part. Error bars are 95\% CIs.
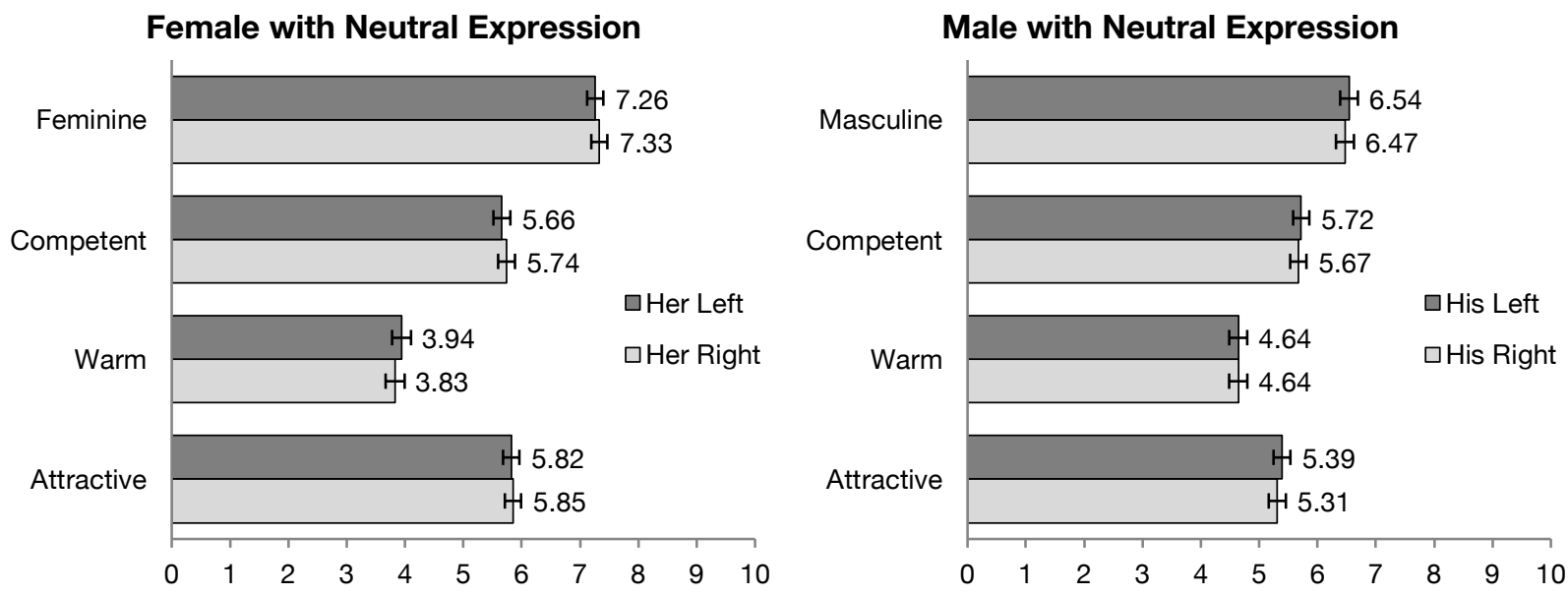

Female with Smiling Expression
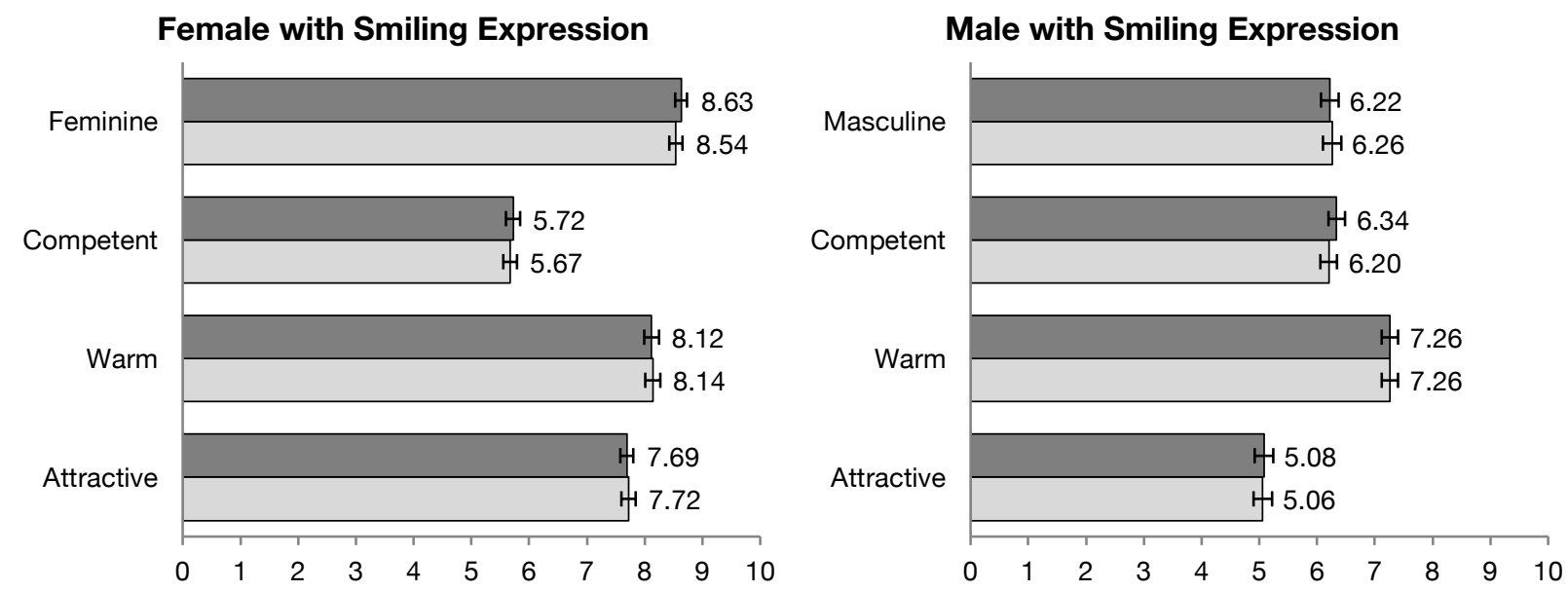
Table 1. Null hypothesis and Bayesian statistics from 2 (Model: Male, Female) $\times 2$ (Expression: Neutral, Smiling) $\times$ 4 (Trait: Attractive, Warm, Competent, Gender-Consistent) $\times 2$ (Part Direction: Left, Right) Mixed Model ANOVAs for each of the four traits. Part direction did not alter appearances in general. And none of the interactions with part direction approached significance. In contrast, all effects of model, expression, trait, and their interactions were significant.

\begin{tabular}{rcccc}
\hline & \multicolumn{2}{c}{ Null Hypothesis Testing } & \multicolumn{1}{c}{ Bayesian } \\
\cline { 2 - 3 } & $\boldsymbol{F}$ & $\boldsymbol{p}$ & $\boldsymbol{\eta}_{\mathbf{p}}{ }^{2}$ & $\boldsymbol{p}_{\text {BiC }}\left(\mathbf{H}_{\mathbf{0}} \mid \mathbf{D}\right)$ \\
Model & 971.69 & $<.001$ & .375 & \\
Expression & 2457.26 & $<.001$ & .603 & \\
Trait & 442.67 & $<.001$ & .215 & \\
Part Direction & 0.11 & .74 & $<.001$ & .985 \\
Model $\times$ Expression & 1377.67 & $<.001$ & .460 & \\
Model $\times$ Trait & 303.75 & $<.001$ & .158 & \\
Model $\times$ Part Direction & 0.54 & .46 & $<.001$ & .964 \\
Expression $\times$ Trait & 1017.73 & $<.001$ & .386 & \\
Expression $\times$ Part Direction & 0.01 & .91 & $<.001$ & .988 \\
Trait $\times$ Part Direction & 0.02 & .99 & $<.001$ & $>.999$ \\
Model $\times$ Expression $\times$ Trait & 26.09 & $<.001$ & .016 & \\
Model $\times$ Expression $\times$ Part Direction & 0.19 & .66 & $<.001$ & .974 \\
Expression $\times$ Trait $\times$ Part Direction & 0.59 & .62 & $<.001$ & $>.999$ \\
Model $\times$ Expression $\times$ Trait $\times$ Part Direction & 1.98 & .12 & .001 & $>.999$ \\
\hline
\end{tabular}

Might the failure to detect an effect of hair part on appearance be a product of participants not paying attention? If so, then I would expect other appearance factors (such as smiling) to also yield null results. However, Table 1 shows that every other factor in the omnibus reached statistical significance, and six of seven had very large effect sizes. For example, smiling had a sizeable impact on the models' appearance. The failure to detect an effect of hair part on the overall impression does not seem to be explained by participants not paying attention. 
Table 2. Inferential statistics from 2 (Model: Male, Female) $\times 2$ (Expression: Neutral, Smiling) $\times 2$ (Part Direction: Left, Right) Mixed Model ANOVAs for each of the four traits.

\begin{tabular}{|c|c|c|c|c|c|c|c|c|}
\hline & \multicolumn{2}{|c|}{ Attractive } & \multicolumn{2}{|c|}{ Warm } & \multicolumn{2}{|c|}{ Competent } & \multicolumn{2}{|c|}{ Gender-Consistent } \\
\hline & $\boldsymbol{F}$ & $\eta_{\mathrm{p}}^{2}$ & $\boldsymbol{F}$ & $\eta_{p}^{2}$ & $\boldsymbol{F}$ & $\eta_{p}^{2}$ & $\boldsymbol{F}$ & $\eta_{p^{2}}^{2}$ \\
\hline Model & $922.83 * * *$ & .360 & 1.37 & .001 & $276.34 * * *$ & .144 & $861.27 * * *$ & .346 \\
\hline Expression & $460.44 * * *$ & .219 & $2676.19 * * *$ & .620 & $868.19^{* * *}$ & .347 & $249.45 * * *$ & .133 \\
\hline Part Direction & 0.07 & $<.001$ & 0.24 & $<.001$ & 0.15 & $<.001$ & 0.00 & $<.001$ \\
\hline Model $\times$ Expression & $1014.62 * * *$ & .382 & $524.46 * * *$ & .242 & $434.34 * * *$ & .210 & $485.39 * * *$ & .230 \\
\hline Model $\times$ Part Direction & 0.67 & $<.001$ & 0.16 & $<.001$ & $2.92^{\dagger}$ & .002 & 0.00 & $<.001$ \\
\hline Expression $\times$ Part Direction & 0.13 & $<.001$ & 0.22 & $<.001$ & 1.06 & .001 & 0.18 & $<.001$ \\
\hline Model $\times$ Expression $\times$ Part Direction & 0.19 & $<.001$ & 0.89 & .001 & 0.00 & $<.001$ & $3.74^{\dagger}$ & .002 \\
\hline
\end{tabular}

Note. ${ }^{\dagger} p<.10,{ }^{*} p<.05,{ }^{* *} p<.01, * * * p<.001$

Table 3. Contrasts between the left and right hair part for all 16 impression variables.

\begin{tabular}{|c|c|c|c|c|c|c|}
\hline \multirow{2}{*}{$\begin{array}{c}\text { Trait } \\
\text { Female Subject }\end{array}$} & \multicolumn{3}{|c|}{ Neutral Expression } & \multicolumn{3}{|c|}{ Smiling } \\
\hline & $\begin{array}{l}\text { Difference } \\
{[95 \% \mathrm{CI}]}\end{array}$ & $p$ & $d$ & $\begin{array}{l}\text { Difference } \\
{[95 \% \text { CI] }}\end{array}$ & $p$ & $d$ \\
\hline Attractive & $-0.02[-0.22,0.17]$ & .82 & -0.010 & $-0.02[-0.19,0.14]$ & .87 & -0.018 \\
\hline Warm & $0.11[-0.12,0.34]$ & .34 & 0.047 & $-0.02[-0.20,0.17]$ & 1.00 & -0.005 \\
\hline Competent & $-0.10[-0.30,0.10]$ & .34 & -0.048 & $0.00[-0.17,0.17]$ & .33 & 0.000 \\
\hline Feminine & $-0.08[-0.27,0.12]$ & .43 & -0.040 & $0.08[-0.08,0.23]$ & .38 & 0.044 \\
\hline \multicolumn{7}{|l|}{ Male Subject } \\
\hline Attractive & $0.09[-0.11,0.30]$ & .38 & 0.043 & $0.03[-0.19,0.26]$ & .78 & 0.013 \\
\hline Warm & $0.01[-0.21,0.23]$ & .91 & 0.009 & $0.01[-0.19,0.21]$ & .94 & 0.000 \\
\hline Competent & $0.05[-0.14,0.25]$ & .59 & 0.025 & $0.15[-0.06,0.35]$ & .15 & 0.067 \\
\hline Masculine & $0.07[-0.15,0.28]$ & .55 & 0.032 & $-0.04[-0.26,0.18]$ & .72 & -0.018 \\
\hline
\end{tabular}




\section{Discussion}

A high-powered experiment failed to detect any effect of hair part orientation on a portrait's appearance, regardless of whether the subject was female or male, or had a neutral or smiling expression. In contrast, impressions varied with every other factor involved (i.e. the model, perceived trait, expression, and their interactions), meaning that hair part was unique in its non-relation to impression. These results provisionally suggest that hair part has little or no effect on appearance. To reach that conclusion requires interpreting a null effect. A limitation of Study 1 was the absence of positive evidence that hair part does not alter appearance. I aimed to address that in Study 2.

\section{Study 2}

Study 2 leveled the conceptual playing field between the hypothesis that one hair part is better than the other and the hypothesis that there is no difference, and tested whether one was more supported. Participants simultaneously viewed a left part and an analogous right part portrait, side-by-side. If hair part does not alter appearance, the modal response should be to deny a question about which one looks better by indicating that neither is better. I also tested whether the belief that hair part matters is widespread.

\section{Method}

Participants. Participants were 913 Americans on AMT (51\% male, $M=37$ years old, $S D=11$, range 18-73), recruited the same way as in Study 1.

Procedure. Participants indicated which of two portraits they preferred, indicated their own preferred hair part for themselves, and reported demographics.

Judgments of portraits. Participants viewed two portraits of a female model (see Figure 1). The two portraits were side-by-side. In one portrait, her hair part was on her left. In the other 
portrait, her hair part was on her right. Participants responded to the question, "Which of these looks better?" by selecting one or the other image, or by indicating "neither/same." Given the leading nature of question and the obvious difference in the two portraits, the demand characteristic was likely to select one or the other portrait, stacking the odds against finding evidence that they are the same. Participants viewed both the neutral facial expression portraits and the smiling portraits, with the order of presentation counterbalanced. The placement of the portraits (i.e. the left hair part to the left or right of the right hair part) was also counterbalanced.

Judgments of self. Participants reported their belief about their own hair part. The question asked, "Does it look better when you part your hair on your left or on your right?" Response options were "Better on my left”, "Better on my right", "Exactly the same”, and “Unsure/Don't care". Because participants who selected the final option $(n=208)$ may not have a hair part (or hair for that matter), I treated their responses on this item as missing data.

\section{Results}

Judgments of portraits. When viewing the two portraits of the same subject with a neutral expression and a hair part on the left or right, the numbers of participants who select the left part, right part, and same/neither options was not evenly distributed, $\chi^{2}(2, N=911)=107.25$, $p<.001, \varphi=.343$. Table 4 shows how participants who indicated that the right part or left part was superior were under-represented, whereas participant who indicated that they look the same were over-represented. I found the same general pattern with the smiling expression portraits, $\chi^{2}$ $(2, N=911)=33.41, p<.001, \varphi=.192($ see Table 4$)$, wherein people who indicated that there was no clear winner were over-represented, and participants who preferred the left part were under-represented. These results favor the hypothesis that there is no difference between the two. 
Comparing only those participants that indicated a preference for the left or right hair part, I found no bias on the neutral expression, $z=0.14, p=.59$, relative risk $=0.99$, and a small bias favoring the right part when the subject was smiling, $z=2.35, p=.009$, relative risk $=1.10$. These results need to be interpreted cautiously as they ignore the modal response, that there is no difference between the two portraits.

Table 4. Proportions of participants that preferred the left or right hair part, or expressed no preference, along with statistics testing whether the proportion differs from chance $(33.3 \%)$.

\begin{tabular}{|c|c|c|c|c|c|c|c|c|}
\hline & \multicolumn{4}{|c|}{$\begin{array}{l}\text { Neutral Expression } \\
\quad(n=911)\end{array}$} & \multicolumn{4}{|c|}{$\begin{array}{l}\text { Smiling } \\
(n=907) \\
\end{array}$} \\
\hline & Observed & $\mathrm{RR}$ & $z$ & $p$ & Observed & RR & $z$ & $p$ \\
\hline Right Part & $25.0 \%$ & 0.75 & -5.28 & $<.001$ & $32.2 \%$ & 0.97 & -0.69 & .245 \\
\hline No Difference & $49.5 \%$ & 1.49 & 10.32 & $<.001$ & $41.7 \%$ & 1.25 & 5.29 & $<.001$ \\
\hline Left Part & $25.5 \%$ & 0.77 & -5.00 & $<.001$ & $26.1 \%$ & 0.78 & -4.57 & $<.001$ \\
\hline
\end{tabular}

Note. $\mathrm{RR}=$ relative risk.

Judgments of self. In contrast to judgments of the subject (which favored the no difference hypothesis) participants indicated a preference for one or the other hair part when it came to their own appearance. Table 5 presents the results. The responses were not evenly distributed across the three options, $\chi^{2}(2, N=705)=39.05, p<.001, \varphi=.235$. Right and left part preferences were over-represented, and the "no difference" response was under-represented. Comparing only those participants that indicated a preference for parting their hair on the left or right, responses were evenly distributed, $z=1.33, p=.185$, relative risk $=0.94$. (Gender did not moderate this general non-preference, $\left.\chi^{2}(2, N=682)=2.67, p=.264, \varphi=.063\right)$.

To explore the relationship between judgments of others and judgments of the self, I coded left part preference as -1 , no difference as 0 , and right part preference as 1 , and examined the correlation between preferences for self versus others. Own part preference was unrelated to preference in others with a neutral expression, $r(701)=-.030, p=.431$, and with a smiling 
expression, $r(697)=.002, p=.959$, meaning that preferences for one's own hair part did not generalize to judgments of others.

Table 5. Proportions of participants that indicated their own hair looks better when parted on their left or right, along with statistics testing whether the proportion differs from chance (33.3\%).

\begin{tabular}{lrrrr} 
& Observed & Relative Risk & $z$ & $p$ \\
\hline Right Part & $36.5 \%$ & 1.09 & 1.72 & .043 \\
No Difference & $22.6 \%$ & 0.68 & -6.19 & $<.001$ \\
Left Part & $41.0 \%$ & 1.23 & 4.27 & $<.001$ \\
\hline
\end{tabular}

\section{Discussion}

Leveling the conceptual playing field between Hair Part Theory and the No Difference hypothesis yielded evidence supporting the latter. However, even after the modal participant indicated that there is no difference between the left and right hair part when viewing others, most participants still indicated a preference for one hair part over the other for themselves. This observation may reflect an enduring cultural belief that hair part matters.

\section{Study 3}

A limitation of the previous studies is that they uniformly relied on models with their hair parted on their left by default. This, and the inclusion of just two models, limits the generalizability of the result. In Study 3, I used four new models, all with their hair parted on their right in the original portraits. A second limitation of the previous studies was the use of portraits in which the hair flowed with the hair whorl. A hair whorl is the natural clockwise or counterclockwise directionality of the hair flow from the scalp. When people part their hair against the whorl, the hair tends to stand up higher. It remains possible that the left hair part looks better than the right (or vice versa) when the hair flows against the whorl. In Study 3, I included models whose part flowed with or against their hair whorl before digitally reversing the hair part. 


\section{Method}

Participants. I recruited $N=1252$ Americans from Amazon's Mechanical Turk (AMT; each received $\$ 0.20)$. The sample spanned much of the lifespan (18-80 years) with $M=36.4$ (SD $=11.6)$, and was gender-balanced (55\% male, $45 \%$ female).

Procedure. The procedure was identical to that of Study 1, except for the portraits and character traits. For each participant, the hair part in all four images was on the left or on the right (randomly assigned between-subjects; $n \mathrm{~s}=613 \& 639$, respectively). The stimuli were four new individuals from the Chicago Faces Database (Ma et al., 2015; see Figure 4). They depicted a White female or White male model, with the hair part flowing either with or against the person's natural hair whorl. I ascertained hair whorls by closely inspecting the orientation of the hair as it exited the head above the forehead. Unlike in Studies 1 and 2, all of the original images had the hair part on the model's right. I again digitally altered the hair part to create the right part images.

Participants rated each image on six character traits, with three representing the overarching dimension of warmth/communion (moral, interdependent, feminine) and three representing competence/agency (competent, independent, masculine). The items were from Abele and Wojciszke (2007) and the scales were anchored at 0 (not at all), 25 (slightly), 50 (moderately), 75 (very much), and 100 (extremely). The reliability of the aggregates was somewhat low: the warmth scale's $\alpha$ ranged from .28-.70, with $M=.46$, and the competence scales' $\alpha$ ranged from .26-.49, with $M=.35$. So I analyzed each item separately. 
Figure 4. Experimental stimuli (Study 3). Participants saw four pictures of a male and female model with their hair parted against (top row) or with (bottom row) their hair whorls. I randomly assigned each participant to see the hair part on the left or the right in all four portraits. The order of presentation was counterbalanced.

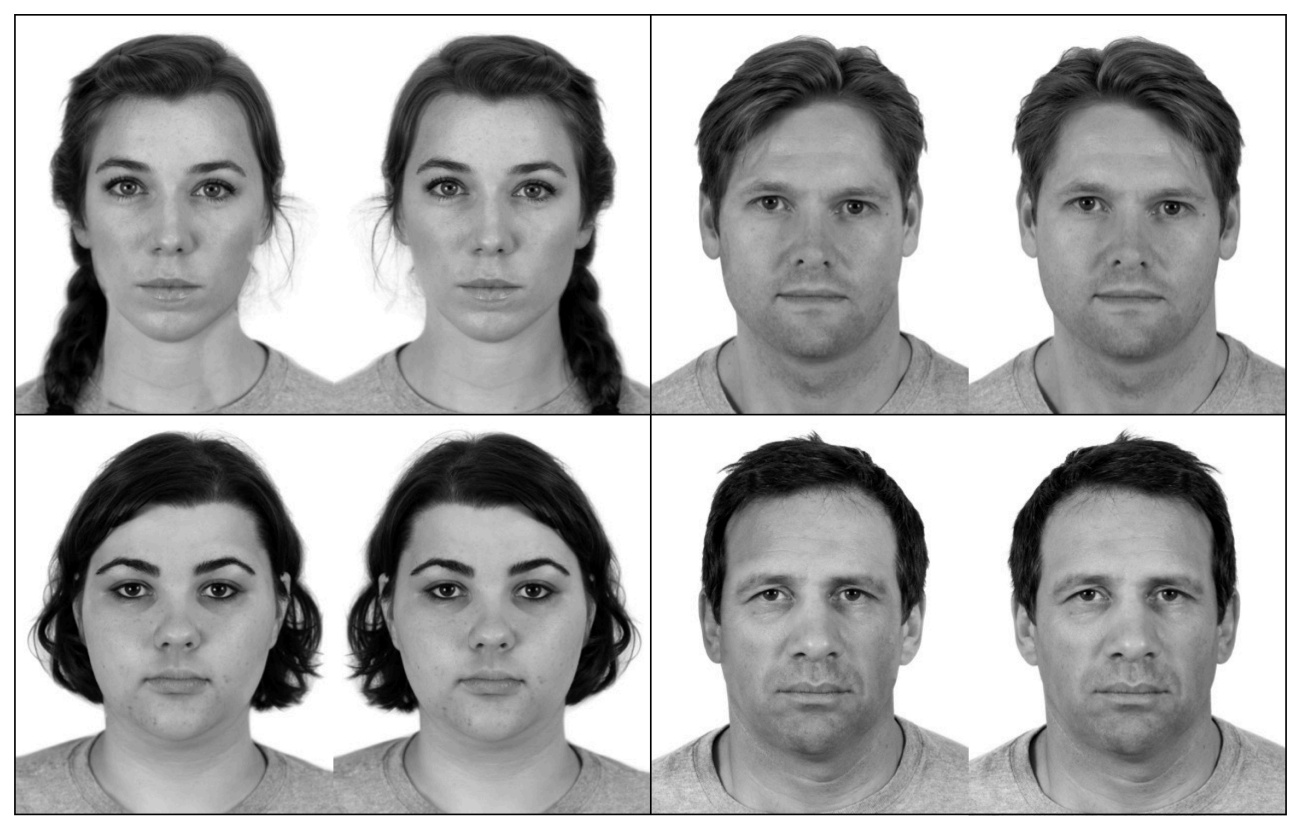

\section{Results}

Once again, hair part orientation had a negligible effect on the rated character of the portrayed models (see Figure 5). Table 6 presents the results of a 2 (Model Gender: Male, Female $) \times 2$ (Flow: Parted against or with the Whorl) $\times 4$ (Trait: Moral, Interdependent, Feminine, Competent, Independent, Masculine $) \times 2$ (Part Direction: Left, Right) Mixed Model ANOVA (with the first three factors within-subjects and the final factor between-subjects). Like in Study 1, hair part orientation did not alter the appearance of the models, regardless of the model's gender, hair flow, or trait, or any combination thereof. All effects involving hair part had $>96 \%$ probability of the null being true given the data. All effects not involving hair part were again significant and most had very large effect sizes, suggesting that participants were paying attention. 
Figure 5. Rating of the female and female models with the hair flow parted with or against their whorls, and with the hair part on their left or right. In spite of the large sample size and statistical power, I failed to detect any significant variance associated with hair part. Error bars are 95\% CIs.

Female; Parted Against Whorl

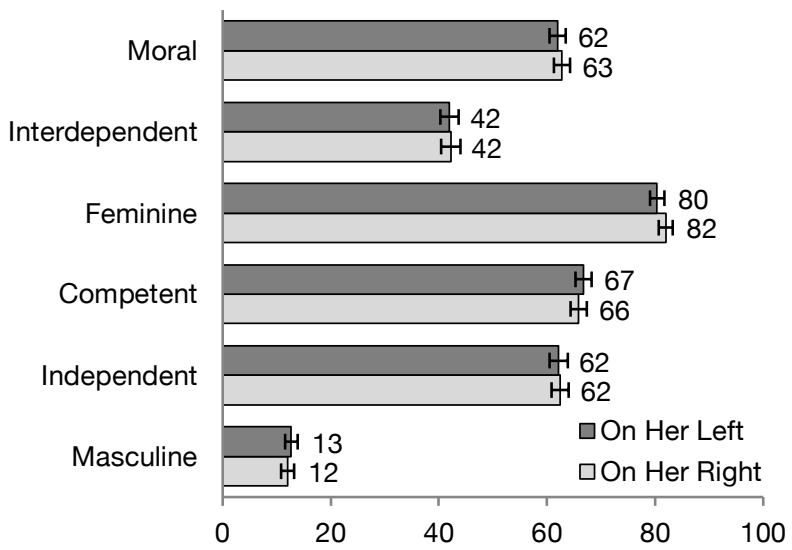

Female; Parted With Whorl

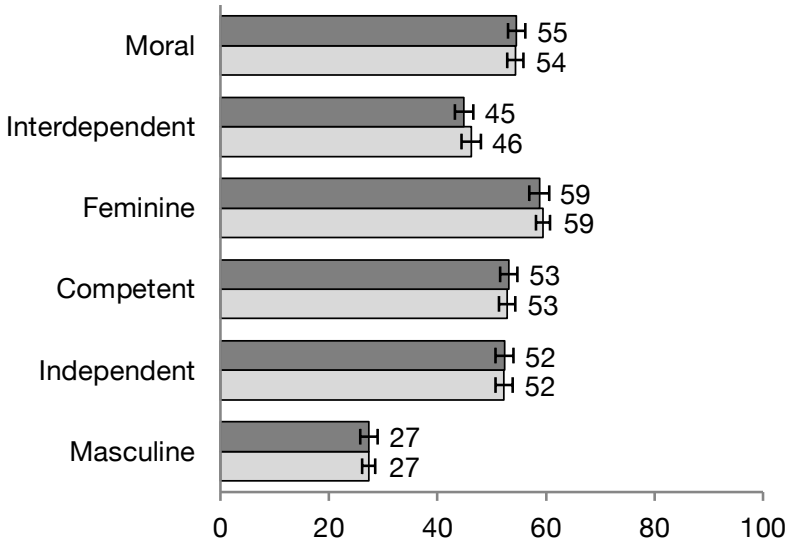

Male; Parted Against Whorl

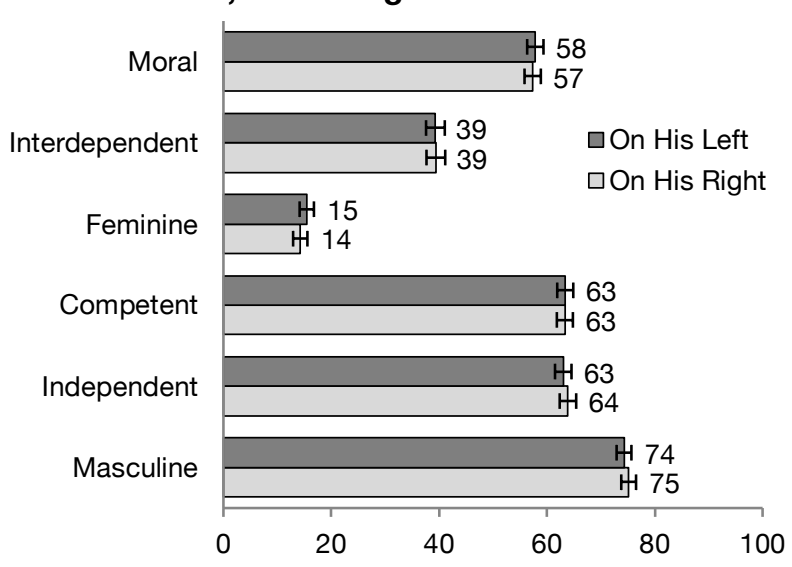

Male; Parted With Whorl

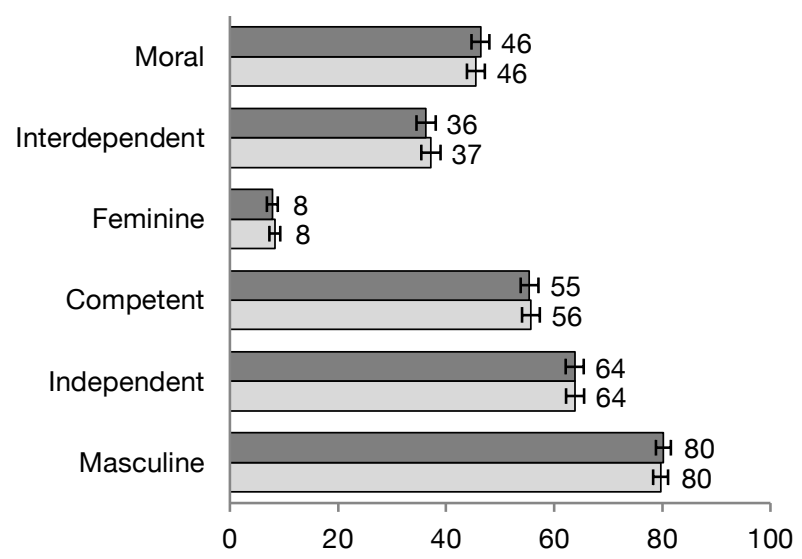


Table 6. Null hypothesis and Bayesian statistics from 2 (Model Gender: Male, Female) $\times 2$ (Flow: Parted against or with the Whorl) $\times 4$ (Trait: Moral, Interdependent, Feminine, Competent, Independent, Masculine) $\times 2$ (Part Direction: Left, Right) Mixed Model ANOVAs. Part direction did not alter appearances in general. And none of the interactions with part direction approached significance. In contrast, all other effects were significant.

\begin{tabular}{|c|c|c|c|c|}
\hline & \multicolumn{3}{|c|}{ Null Hypothesis Testing } & \multirow{2}{*}{$\begin{array}{c}\text { Bayesian } \\
p_{\text {BIC }}\left(\mathbf{H}_{0} \mid \mathrm{D}\right)\end{array}$} \\
\hline & $F$ & $p$ & $\eta_{p}^{2}$ & \\
\hline Gender & 33.76 & $<.001$ & .027 & \\
\hline Flow & 604.64 & $<.001$ & .334 & \\
\hline Trait & 729.40 & $<.001$ & .377 & \\
\hline Part Direction & 0.07 & .80 & $<.001$ & .971 \\
\hline Gender $\times$ Flow & 29.50 & $<.001$ & .024 & \\
\hline Gender $\times$ Trait & 4432.59 & $<.001$ & .786 & \\
\hline Gender $\times$ Part Direction & 0.20 & .66 & $<.001$ & .969 \\
\hline Flow $\times$ Trait & 412.46 & $<.001$ & .255 & \\
\hline Flow $\times$ Part Direction & 0.01 & .94 & $<.001$ & .972 \\
\hline Trait $\times$ Part Direction & 0.30 & .91 & $<.001$ & $>.999$ \\
\hline Gender $\times$ Flow $\times$ Trait & 153.08 & $<.001$ & .113 & \\
\hline Gender $\times$ Flow $\times$ Part Direction & 0.00 & .96 & $<.001$ & .972 \\
\hline Flow $\times$ Trait $\times$ Part Direction & 0.57 & .73 & $<.001$ & $>.999$ \\
\hline Gender $\times$ Flow $\times$ Trait $\times$ Part Direction & 0.88 & .49 & .001 & $>.999$ \\
\hline
\end{tabular}

\section{Discussion}

Study 3 used portraits from four new models and once again found that hair part did not alter the person's appearance. The inclusion of portraits in which the original hair part was on the right, and the hair part flowed with or against the individual's hair whorl, enhanced the generalizability of the findings from Studies 1 and 2.

\section{General Discussion}

Study 1 and 3 tested whether hair part alters a person's appearance. In spite of the large statistical power, the study failed to yield evidence that hair part matters, and Bayesian analyses favored the null. Study 2 supplied positive evidence that hair part does not alter appearance. The three studies included six different models. Their original hair part was on the left or right, they had smiling or neutral expressions, and their hair part flowed with or against their natural hair 
whorl. Together, the findings challenge the conventional wisdom that the side of the head on which one parts their hair affects the person's appearance.

A noteworthy design feature of the present studies was portraits in which the hair part was digitally reversed without alteration to the hairstyle or facial expression. The strength of this design feature is that it isolated the effect of hair part orientation on appearance. Although Study 3 did include portraits in which the hair flowed either with or against the whorl, an associated weakness of this design is that the studies do not permit clear conclusions regarding whether switching one's hair part alters a person's appearance. Switching a hair part from left to right or vice versa would involve changing whether the hair flows with or against the hair whorl. Thus, switching hair parts would change the hair part orientation (left, right), and often change the hairstyle (e.g., its thickness). If switching the hair part does change a person's appearance, the results of the present studies suggest that it would probably be because of the change in hairstyle, and probably not because of the hair part orientation.

The present studies also cannot speak to whether hair part matters in color portraits, in moving images, or in real life. Results from the studies do not generalize to non-White models, or in countries other than the U.S. And the use of six models limits the generalizability of the findings to these six individuals. Perhaps for some individuals, facial asymmetries such as the distinctive moles, scars, or tattoos, or even structural asymmetries in the face or hair, could make the left hair part look better or worse than the right. These limitations notwithstanding, these studies are the first to test the popular cultural belief that hair part matters in general. The results favor the view that the popular belief is either a myth of underspecified. 


\section{References}

Abele, A. E., \& Wojciszke, B. (2007). Agency and communion from the perspective of self versus others. Journal of Personality and Social Psychology, 93(5), 751-763. doi: $0.1037 / 0022-3514.93 .5 .751$

Abele, A. E., \& Wojciszke, B. (2013). The Big Two in social judgment and behavior. Social Psychology, 44(2), 61-62. doi:10.1027/1864-9335/a000137

Abumrad, J. \& Krulwich, R. (n.d.). Mirror, mirror. Radiolab. Retrieved from http://www.radiolab.org/story/122613-mirror-mirror/

Borod, J. C., Kent, J., Koff, E., Martin, C., \& Alpert, M. (1988). Facial asymmetry while posing positive and negative emotions: Support for the right hemisphere hypothesis. Neuropsychologia, 26(5), 759-764. doi:10.1016/0028-3932(88)90013-9

Borod, J. C., Koff, E., \& White, B. (1983). Facial asymmetry in posed and spontaneous expressions of emotion. Brain and Cognition, 2(2), 165-175. doi:10.1016/02782626(83)90006-4

Campbell, R. (1978). Asymmetries in interpreting and expressing a posed facial expression. Cortex, 14(3), 327-342. doi:10.1016/S0010-9452(78)80061-6

Cuddy, A. C., Fiske, S. T., \& Glick, P. (2007). The BIAS map: Behaviors from intergroup affect and stereotypes. Journal of Personality and Social Psychology, 92(4), 631-648. doi:10.1037/0022-3514.92.4.631

Ekman, P., Hager, J. C., \& Friesen, W. V. (1981). The symmetry of emotional and deliberate facial actions. Psychophysiology, 18(2), 101-106. doi:10.1111/j.14698986.1981.tb02919.x

Ellerson, L. (2008, July 18). Hillary Clinton and the Hair Part Theory. ABC News. Retrieved 
from http://blogs.abcnews.com/politicalpunch/2008/07/hillary-clinton.html

Female Magazine (2013, August 15). What does you hair parting say about you? Female.

Retrieved from http://femalemag.com.my/beauty/what-does-your-hair-parting-say-aboutyou/

Indersmitten, T., \& Gur, R. C. (2003). Emotion Processing in Chimeric Faces: Hemispheric Asymmetries in Expression and Recognition of Emotions. The Journal of Neuroscience, 23(9), 3820-3825.

Ma, D. S., Correll, J., Wittenbrink, B. (2015). The Chicago face database: A free stimulus set of faces and norming data. Behavior Research Methods, 47, 1122-1135.

Masson, M. E. J. (2011). A tutorial on a practical Bayesian alternative to null-hypothesis significance testing. Behavioral Research Methods, 43(3), 679-690. Doi: $10.3758 / \mathrm{s} 13428-010-0049-5$

Murphy, C. (1999, June). The mirror of Dorian Gray: Mirrors never lie, they say. But how much truth do we really want? The Atlantic. Retrieved from https://www.theatlantic.com/magazine/archive/1999/06/the-mirror-of-doriangray/377630/

Nicholls, M. E., Ellis, B. E., Clement, J. G., \& Yoshino, M. (2004). Detecting hemifacial asymmetries in emotional expression with three-dimensional computerized image analysis. Proceedings of the Royal Society B: Biological Sciences, 271 (1540), 663-338. doi: $10.1098 / \mathrm{rspb} .2003 .2660$

Rosenberg, S., Nelson, C., \& Vivekananthan, P. S. (1968). A multidimensional approach to the structure of personality impressions. Journal of Personality and Social Psychology, 9(4), 283-294. doi:10.1037/h0026086 
Rubin, D. A., \& Rubin, R. T. (1980). Differences in asymmetry of facial expression between left- and right-handed children. Neuropsychologia, 18(3), 373-377. doi:10.1016/00283932(80)90135-9

Sackeim, H. A., \& Gur, R. C. (1978). Lateral asymmetry in intensity of emotional expression. Neuropsychologia, 16(4), 473-481. doi:10.1016/0028-3932(78)90070-2

Sackeim, H. A., Gur, R. C., \& Saucy, M. C. (1978). Emotions are expressed more intensely on the left side of the face. Science, 202(4366), 434-436. doi:10.1126/science.705335

Schillinger, L. (2001, January 29). Optical delusions: Al Gore's hair problem. The New Yorker. Retrieved from https://www.newyorker.com/magazine/2001/01/29/optical-delusions-algores-hair-problem

Skinner, M., \& Mullen, B. (1991). Facial asymmetry in emotional expression: A meta-analysis of research. British Journal of Social Psychology, 30(2), 113-124. doi:10.1111/j.20448309.1991.tb00929.x

Trapnell, P. D., \& Wiggins, J. S. (1990). Extension of the Interpersonal Adjective Scales to include the Big Five dimensions of personality. Journal of Personality and Social Psychology, 59(4), 781-790. doi:10.1037/0022-3514.59.4.781

Walter, J. \& Walter, C. (1999). What is your hair part saying about you? The effects of hair parting on social appraisal and personality development. Unpublished manuscript. Retrieved from https://hairparttheory.files.wordpress.com/2011/05/hairparttheoryc2001tmcinc.pdf 